\title{
Curious behavior of three-dimensional lattice Dirac operators coupled to a monopole background
}

\author{
Nikhil Karthik* \\ Physics Department, Brookhaven National Laboratory, Upton, New York 11973-5000, USA \\ Rajamani Narayanan $\oplus^{\dagger}$ \\ Department of Physics, Florida International University, Miami, Florida 33199, USA
}

(Received 19 August 2019; published 11 November 2019)

\begin{abstract}
We numerically investigate the effect of regulating fermions in the presence of singular background fields in three dimensions. For this, we couple free lattice fermions to a background compact U(1) gauge field consisting of a monopole-antimonopole pair of magnetic charge $\pm Q$ separated by a distance $s$ in a periodic $L^{3}$ lattice and study the low-lying eigenvalues of different lattice Dirac operators under a continuum limit defined by taking $L \rightarrow \infty$ at fixed $s / L$. As the background gauge field is parity even, we look for a twofold degeneracy of the Dirac spectrum that is expected of a continuumlike Dirac operator. The naive-Dirac operator exhibits such a parity doubling but breaks the degeneracy of the fermion-doubler modes for the $Q$ lowest eigenvalues in the continuum limit. The Wilson-Dirac operator lifts the fermion doublers but breaks the parity doubling in the $Q$ lowest modes even in the continuum limit. The overlapDirac operator shows parity doubling of all the modes even at finite $L$ that is devoid of fermion doubling and is singled out as a properly regulated continuum Dirac operator in the presence of singular gauge field configurations, albeit with a peculiar algorithmic issue.
\end{abstract}

DOI: 10.1103/PhysRevD.100.094501

\section{INTRODUCTION}

Lattice regularization of noncompact QED [1] in three dimensions is defined by a noncompact action for the gauge fields, $\theta_{\mu}(\mathbf{n}) \in \mathbf{R}$, on the link connecting $\mathbf{n}$ and $\mathbf{n}+\hat{\mu}$, and the lattice fermions couple to $U(1)$ valued link variables, $U_{\mu}(\mathbf{n})=e^{i \theta_{\mu}(\mathbf{n})}$. Monopoles are suppressed in the continuum limit in such a regularization. Recent numerical analysis of noncompact QED in three dimensions with an even number of massless two-component fermions shows that these theories are scale invariant independent of the number of flavors [2-4]. It is natural to follow up such a study with an analysis of compact $\mathrm{QED}_{3}$ where the lattice gauge action is the compact gauge action [5]. When we attempted to numerically study this theory using overlapDirac fermions, we found it be numerically formidable due to anomalously small eigenvalues of the massive Wilson-Dirac kernel that is at the core of the overlapDirac operator-to contrast, for a smooth field, one would

\footnotetext{
*nkarthik@bnl.gov

rajamani.narayanan@fiu.edu
}

Published by the American Physical Society under the terms of the Creative Commons Attribution 4.0 International license. Further distribution of this work must maintain attribution to the author(s) and the published article's title, journal citation, and DOI. Funded by SCOAP. find the spectrum of a massive Wilson-Dirac operator to be gapped at least by the Wilson mass. This prompted us to consider the question of what happens when the conventional lattice regulated fermions, which lead to universal results in the continuum limit over generic smooth gauge fields, are coupled to a singular gauge field from a monopole; do operations at the level of lattice spacing, such as point splitting used regularly in lattice regularization, have any effect in the presence of a Dirac string which is also one lattice spacing thick? We present related numerical observations in this paper.

Briefly, we recount some aspects of lattice fermions in three dimensions. We use two-component fermions throughout this paper. The naive fermion operator $D$ obtained by using the discrete derivative operator is the simplest. As is well known, it leads to $2^{d}$ ( 8 in three dimensions) fermions flavors. It is a well-motivated expectation that there is flavor degeneracy in the continuum limit. There is a trivial twofold degeneracy for naive-Dirac fermions [6,7] on the lattice, and one copy is the staggered-Dirac fermion, which is expected to realize a four-fermion flavor theory in three dimensions. If there is a fourfold degeneracy in the continuum limit, one could possibly define a theory with the square root of the staggered-Dirac operator to study a two-flavor parity invariant theory. Some continuum-based reasoning provides arguments as to why gauge field backgrounds with 
nontrivial topology might obstruct a well-defined continuum limit of a lattice theory with the fourth root of the staggered-Dirac operator in even dimensions [8-11]. It is possible that monopole backgrounds in three dimensions suffer from similar effects. The two-component WilsonDirac operator is obtained by adding the Wilson term $B$, which is irrelevant by naive power counting, to the naive operator $D$. That is, the massive Wilson-Dirac operator is given by

$$
X=-m_{w}+B+D,
$$

which lifts the mass of the seven of the doublers, leaving only one physical fermion of lattice mass $m_{w}$ on smooth gauge fields. The lattice fermion, which is capable of reproducing the continuum symmetries, such as the $\mathrm{U}(N)$ flavor symmetry in three-dimensional $N$-flavor $\mathrm{QED}_{3}$, is the overlap-Dirac operator. The central quantity that appears in the overlap formalism $[3,12]$ is the unitary operator $V$ defined as

$$
V=X \frac{1}{\sqrt{X^{\dagger} X}},
$$

with the Wilson mass $0<m_{w}<2$, and the massless overlap operator is given by

$$
D_{o}=\frac{1+V}{2} ; \quad D_{o}^{\dagger} D_{o}=\frac{2+V+V^{\dagger}}{4} .
$$

The instance where the otherwise irrelevant operators used in lattice regularization play significant roles is the parity anomaly [13-16]. Parity takes the naive-Dirac operator $D$ to $D^{\dagger}=-D$; the Wilson-Dirac operator $X$ transforms to $X^{\dagger}$, and the unitary operator transforms $V$ to $V^{\dagger}$. The phase of $\operatorname{det} X$ for $m_{w}=0$ is nonvanishing even in the continuum limit, even though the unregulated continuum massless Dirac operator is anti-Hermitian. This effect propagates itself to the nonvanishing phase of $\operatorname{det}(1+V)$ of the massless overlap fermion. Notwithstanding such effects in three dimensions, we expect $X$ to commute with $X^{\dagger}$ in the continuum limit, unless the gauge field background is not smooth even in the continuum limit. Independent of the nature of the gauge field background, $V$ and $V^{\dagger}$ commute. This places the overlap-Dirac operator closer to the continuum Dirac operator compared to the WilsonDirac operator. The domain-wall-Dirac operator formalism in three dimensions $[17,18]$ is expected to behave like the overlap-Dirac operator.

Having explained the lattice formalism, we return back to the problem that motivated us to study the problem to be presented in this paper. Following the conventions of Ref. [3], we will assume that $m_{w}>0$ in the region of interest, and this will lead us to the unconventional notation for Wilson-Dirac fermions; namely, $m_{w}<0$ will correspond to fermions with positive mass. Since the operator $X^{\dagger} X$ can be viewed as the one for two flavors of two-component fermions that preserves parity, the sign of the mass should not matter in the conventional approach to the continuum limit. But our attempts to study compact QED with overlap-Dirac fermions failed due to several eigenvalues of $X^{\dagger} X$ becoming very small for all values of $m_{w} \in(0,2)$. Furthermore, we found the number of such anomalously small eigenvalues to grow with the size of the three-dimensional torus.

The failure described in the previous paragraph prompted us to study the low-lying spectrum of the following positive definite operators constructed out of lattice operators; $D^{\dagger} D$ for the naive-Dirac operator; $X^{\dagger} X$ as a function of $m_{w}$ for the Wilson-Dirac operator; and of the $(1+V)\left(1+V^{\dagger}\right)$ for the overlap-Dirac operator in a controlled background before proceeding to address an alternative approach to the study of compact QED. As we will argue, the eigenvalues of such a positive definite operator is doubly degenerate in the continuum in a monopole-antimonopole background and hence serve as a promising observable to look for any deviation of the regulated lattice operator from the continuum one. It is not possible to write down a background gauge field that has a single monopole in a periodic lattice, but it is possible to write down one that has a monopoleantimonopole pair separated by a fixed distance. Such a background was considered in a study of the monopole scaling dimension [19]. We will use a similar background with a minor change to better fit it in a periodic lattice.

\section{LATTICE MONOPOLE-ANTIMONOPOLE FIELD}

An expression for the standard spherical Dirac monopole with a specific choice for the direction of the semiinfinite Dirac string can be found in Ref. [20]. It is natural for us to pick the direction of the Dirac string along one of the spatial directions on the lattice. Since we will work on a finite periodic lattice, we need to insert a Dirac monopole-antimonopole pair with the string attached between the monopole and the antimonopole. One way to include the monopole-antimonopole background field on the lattice is to integrate the continuum field $\mathcal{A}$ of a Dirac monopole-antimonopole pair [20] over links joining site $\mathbf{x}$ to $\mathbf{x}+a$, where $a$ is the lattice spacing. That is, define a link variable

$$
\bar{\theta}_{\mu}(x)=\int_{\mathbf{x}}^{\mathbf{x}+a \hat{\mu}} d x_{\mu}^{\prime} \mathcal{A}_{\mu}\left(\mathbf{x}^{\prime}\right),
$$

as given in Ref. [19]. The drawback of this approach is that periodicity of lattice forces artificial jumps in the gauge field across the "boundaries." So, we consider a better construction of the field on periodic lattice below. 


\section{A. Monopole-antimonopole field on periodic lattice}

We place the finite Dirac string along a lattice spatial direction. We implement the background gauge field that contains a monopole-antimonopole pair of integer charge $\pm Q$ and separated by a length $s$ on a periodic lattice of length $L$ as defined by the following noncompact field strength $B_{\mu \nu}(\mathbf{n})$ at the lattice site $\mathbf{n}=\left(n_{1}, n_{2}, n_{3}\right)$ :

$$
\begin{aligned}
& B_{23}(\mathbf{n})=B_{31}(\mathbf{n})=0 \text {; }
\end{aligned}
$$

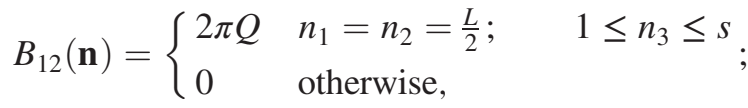

$$
\begin{aligned}
& \mathbf{n} \in[1, L] \text {. }
\end{aligned}
$$

That is, $B_{\mu \nu}(\mathbf{n})$ denotes the noncompact field strength on the directed plaquette defined by the corners $\mathbf{n}, \mathbf{n}+\hat{\mu}$, $\mathbf{n}+\hat{\mu}+\hat{\nu}$, and $\mathbf{n}+\hat{\nu}$ traversed in the counterclockwise direction. We could have spread the jump from 0 to $Q$ and $Q$ to 0 over more than one lattice spacing. This will not change the symmetries, and our choice enables the largest numbers of choices of $L$ without having to go to very large $L$. As constructed, the monopole charge density is

$$
\begin{aligned}
Q(\mathbf{n}) & =\frac{1}{4 \pi} \sum_{\mu \nu \rho} \epsilon_{\mu \nu \rho}\left[B_{\mu \nu}(\mathbf{n}+\hat{\rho})-B_{\mu \nu}(\mathbf{n})\right] \\
& =Q \delta_{n_{1}, \frac{L}{2}} \delta_{n_{2}, \frac{L}{2}}\left[\delta_{n_{3}, 0}-\delta_{n_{3}, s}\right] .
\end{aligned}
$$

As is well known, we cannot find a set of gauge fields, $\theta_{\mu}(\mathbf{n})$, that realizes the above set of plaquette values as their field strength. Instead, one can find a set of gauge fields that minimizes the noncompact action in the presence of a flux background, $B_{\mu \nu}$, given by

$$
\begin{aligned}
S_{g} & =\sum_{\mathbf{n}} \sum_{\mu<\nu=1}^{3}\left[F_{\mu \nu}(\mathbf{n})-B_{\mu \nu}(\mathbf{n})\right]^{2} ; \\
F_{\mu \nu}(\mathbf{n}) & =\theta_{\mu}(\mathbf{n})+\theta_{\nu}(\mathbf{n}+\hat{\mu})-\theta_{\mu}(\mathbf{n}+\hat{\nu})-\theta_{\nu}(\mathbf{n}) .
\end{aligned}
$$

The minimum is easily found by going to the momentum space $\mathbf{k}=\left(k_{1}, k_{2}, k_{3}\right)$ for integer $k_{\mu}$, and the solution is given by

$$
\begin{aligned}
\theta_{\mu}(\mathbf{n}) & =\sum_{\mathbf{k}} \tilde{\theta}_{\mu}(\mathbf{k}) e^{i \frac{2 \pi \mathbf{k} \cdot \mathbf{n}}{L}} ; \quad \tilde{\theta}_{\mu}(\mathbf{k})=\frac{\tilde{J}_{\mu}(\mathbf{k})}{\hat{k}^{2}} ; \\
\hat{k}^{2} & =4 \sum_{\mu=1}^{2} \sin ^{2} \frac{\pi k_{\mu}}{L},
\end{aligned}
$$

where the current is given by

$$
\begin{aligned}
& J_{\mu}(\mathbf{n})=\sum_{\nu}\left[B_{\mu \nu}(\mathbf{n})-B_{\mu \nu}(\mathbf{n}-\hat{\nu})\right] \\
& \tilde{J}_{\mu}(\mathbf{k})=\frac{1}{L^{3}} \sum_{\mathbf{n}} J_{\mu}(\mathbf{n}) e^{-i \frac{2 \pi k \cdot \mathbf{n}}{L}} .
\end{aligned}
$$

The current has no zero momentum component, and the conservation of the current on the lattice is given by $\sum_{\mu}\left[J_{\mu}(\mathbf{n})-J_{\mu}(\mathbf{n}-\hat{\mu})\right]=0$.

\section{B. Parity invariance of the field}

Using the field $\mathcal{A}$ from a continuum Dirac-monopole pair, it is easy to show that the field is parity invariant under $\mathbf{x} \rightarrow-\mathbf{x}$ about the midpoint of the Dirac string connecting the monopole and antimonopole. In order to demonstrate this for the background field as defined above, let us first define the parity operator $P$ via its action $\mathbf{n} \rightarrow \mathbf{n}^{p}=\mathbf{L}-\mathbf{n}$, where $\mathbf{L}=(L, L, L)$. The action of parity on gauge fields on the lattice is then

$$
(P \theta)_{\mu}(\mathbf{n})=\theta_{\mu}^{p}(\mathbf{n})=-\theta_{\mu}(\mathbf{L}-\mathbf{n}-\mu),
$$

and the plaquette defined in Eq. (7) satisfies

$$
F_{\mu \nu}^{p}(\mathbf{n})=F_{\mu \nu}(\mathbf{L}-\mathbf{n}-\hat{\mu}-\hat{\nu}) .
$$

Under this relation, the background flux defined in Eq. (5) satisfies the property

$B_{\mu \nu}^{p}(\mathbf{n}-\mathbf{t})=B_{\mu \nu}(\mathbf{n}) ; \quad \mathbf{t}=(-1,-1, s+1-L)$.

Therefore, the background field that minimizes Eq. (7) will satisfy the property

$$
\theta_{\mu}^{p}(\mathbf{n}-\mathbf{t})=\theta_{\mu}(\mathbf{n})
$$

Let us define the special translation operator $\tau_{\mathbf{t}}$ by

$$
\left[\tau_{\mathbf{t}} \psi\right](\mathbf{n})=\psi(\mathbf{n}+\mathbf{t})
$$

and the standard covariant translation operator $T_{\mu}$ by

$$
\left(T_{\mu}^{\theta} \psi\right)(\mathbf{n})=e^{i \theta_{\mu}(\mathbf{n})} \psi(\mathbf{n}+\hat{\mu}) .
$$

Since

$$
P T_{\mu}^{\theta} P=T_{\mu}^{\theta^{p} \dagger} \quad \text { and } \quad \tau_{\mathbf{t}}^{\dagger} T_{\mu}^{\theta^{p}} \tau_{\mathbf{t}}=T_{\mu}^{\theta},
$$

we arrive at

$$
T_{\mu}^{\theta^{\dagger}}=\bar{P} T_{\mu}^{\theta} \bar{P}^{\dagger} ; \quad \bar{P}=P \tau_{\mathrm{t}} ; \quad \bar{P}^{\dagger} \bar{P}=\mathbf{I} .
$$




\section{Defining continuum limit of the background field}

The continuum limit of a lattice field theory is a subtle limit along the lines of constant physics near a fixed point of the lattice theory. However, in this paper, we consider a comparatively trivial continuum limit-it is possible to define a continuum limit of a background gauge field in such a way that length scales associated with the background field remain fixed with respect to the lattice size. In other words, we set the physical size of the periodic box to be unity by definition and measure all other length scales with respect to it, in which case the lattice spacing is $1 / L$. For example, we can consider a wavelike lattice gauge field $\theta_{\mu}^{\text {wave }}(x)=c_{\mu} / L \cos (2 \pi / L)$ of which the continuum limit $L \rightarrow \infty$ is taken at a fixed value of parameter $c_{\mu}$. In the case of the monopole-antimonopole pair, the associated length scale is the lattice distance $s$ between the monopole and antimonopole. Therefore, we define the continuum limit as the $L \rightarrow \infty$ limit at a fixed value of $f=s / L$. In this paper, we set $f=1 / 4$. Now, it makes sense to ask whether different lattice discretization of the continuum Dirac operator gives universal results in the above defined continuum limit.

It is possible to demonstrate the nontrivial nature of the monopole background that is discretized on the lattice by using the spherical Dirac monopole field $\mathcal{A}$. Since $\mathcal{A}$ is scale invariant, it easy to see that the corresponding lattice field $\bar{\theta}_{\mu}(\mathbf{n})$ that connects the lattice site $\mathbf{n}$ to $\mathbf{n}+\hat{\mu}$ remains invariant at fixed $\mathbf{n}$ for all values of $L$ under the above continuum limit. The reason is the following: when the lattice spacing is reduced by a factor $k$, the physical distance of a lattice site from the monopole reduces by a factor $k$, and hence the physical gauge field at the lattice site increases by a factor $1 / k$. When integrated over a lattice spacing to obtain $\bar{\theta}$, the factor $k$ gets canceled. This is unlike the smooth background $\theta^{\text {wave }}$ considered above, which approaches zero as $1 / L$ in the continuum limit.

The latticelike nature of the background field even in the $L \rightarrow \infty$ limit can be seen in the scaling of the noncompact action $S_{b}=\sum_{\mathbf{n}} \sum_{\mu>\nu} F_{\mu \nu}(\mathbf{n})^{2}$ with $L$ for $F_{\mu \nu}$ obtained through the minimization of Eq. (7). The background field does not have a continuum limit in the usual sense where we expect $\theta_{\mu}(\mathbf{n})$ to be of order $\frac{1}{L}$ and the derivatives to be order $\frac{1}{L}$. In that case, the average value of the action, namely,

$$
p(L)=\frac{1}{3 L^{3}} S_{b}(L)
$$

is expected to go like $\frac{1}{L^{4}}$. Instead, we find that

$$
p(L)=3.271 \frac{1}{L^{2}}-5.85 \frac{1}{L^{3}}
$$

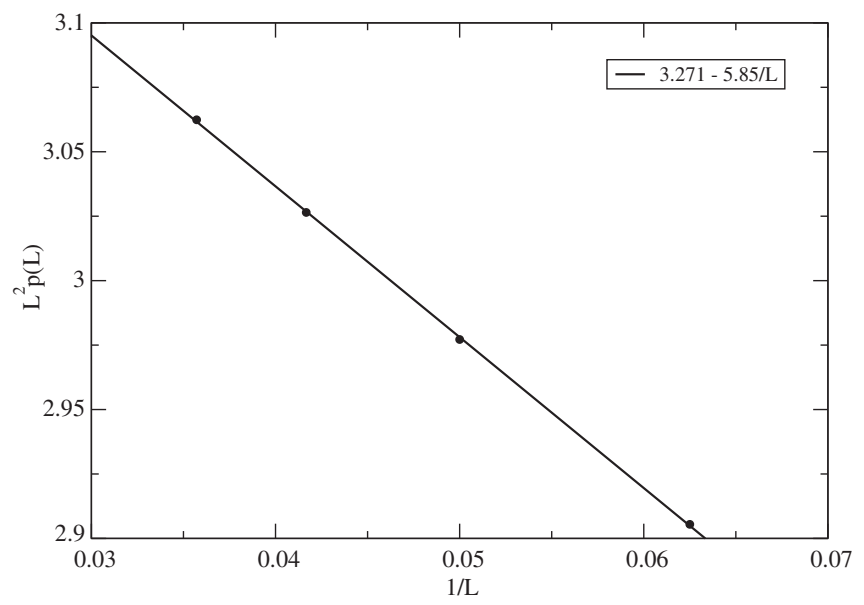

FIG. 1. The action of the background gauge field as a function of $L$.

for the background field discussed in Sec. II A with $Q=1$ as shown in Fig. 1. This atypical behavior is expected due to the presence of a monopole-antimonopole pair in the background gauge field corresponding to singularities in the flux distribution. In the following sections, we will study the effect of this on the low-lying spectrum of fermions.

\section{Parity doubling of continuum Dirac spectrum as reference for lattice fermions}

In order to investigate the effect of the singular nature of the monopole-antimonopole background field on lattice regulated fermions, we need to choose an appropriate observable that is characteristic of the field and has a well-defined property in the unregulated continuum Dirac operator. As we noted above, a characteristic feature of the background field is its parity invariance. For the continuum Dirac operator,

$P D^{\text {cont }}(A) P=-D^{\text {cont }}\left(A^{p}\right) ; \quad D^{\text {cont }}(A)=\not D+i \not A$,

with $A_{\mu}^{p}(\mathbf{x})=-A_{\mu}(-\mathbf{x})$. For parity invariant fields, $A_{\mu}^{p}(\mathbf{x})=A_{\mu}(\mathbf{x})$ up to a gauge transformation. This implies the anticommuting relation

$$
P D^{\text {cont }}(A) P=-D^{\text {cont }}(A) \text {. }
$$

Since $D^{\text {cont }}$ is anti-Hermitian, the above anticommutation property implies that, if $\psi_{+}$is an eigenvector with

$$
D^{\text {cont }} \psi_{+}=i \lambda \psi_{+},
$$

then $\psi_{-}=P \psi_{+}$is an eigenvector with eigenvalue $-i \lambda$. It is convenient to recast this as a statement about $\left(D^{\text {cont }}\right)^{\dagger} D^{\text {cont. }}$

$$
\left[\left(D^{\text {cont }}\right)^{\dagger} \not^{\text {cont }}\right] \psi_{ \pm}=\lambda^{2} \psi_{ \pm} .
$$


Thus, there a parity doubling of eigenvalues of $\left(D^{\text {cont }}\right)^{\dagger} D^{\text {cont }}$. As we will see, the low-lying eigenvalues of $\left(D^{\text {cont }}\right)^{\dagger} D^{\text {cont }}$ and their expected parity doubling lead to unexpected observations for lattice fermions.

The following will then be our method. We will study the low-lying eigenvalue spectrum of lattice Dirac operators in the limit $(L, s) \rightarrow \infty$ at a fixed $f=\frac{s}{L}$. Precisely, we will study the microscopic eigenvalues of the positive definite operator $\left(D^{\text {lat }}\right)^{\dagger} D^{\text {lat }}$ constructed out of the lattice Dirac operators $D^{\text {lat }}$ for the naive-Dirac, Wilson-Dirac, and overlap-Dirac lattice operators in the above background and analyze the low-lying spectrum as a function of $L$ at a fixed $Q$ and $f$. We will mainly consider $Q=1$, and we will set $f=\frac{1}{4}$. We will work with $L$ that are multiples of 4 from $L=12$ to $L=56$. At the end, we will study Wilson-Dirac fermions with $Q=2$ in order to make some conclusions about the study of compact QED using Wilson-Dirac and overlap-Dirac fermions.

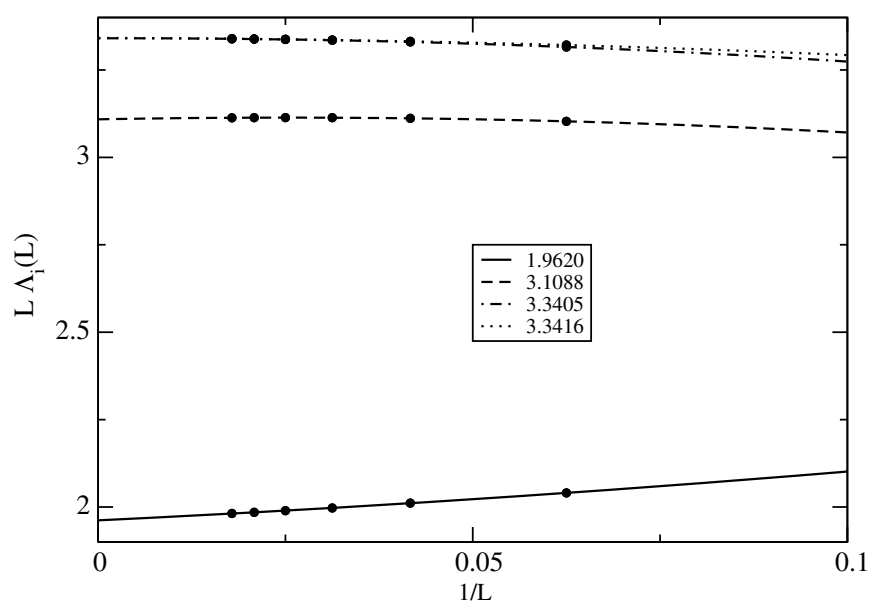

\section{NAIVE-DIRAC FERMIONS}

The naive massless Dirac operator in three dimensions is explicitly given by

$$
\not D=\frac{1}{2} \sum_{\mu=1}^{3} \sigma_{\mu}\left(T_{\mu}-T_{\mu}^{\dagger}\right) ; \quad\left(T_{\mu} \phi\right)(\mathbf{n})=e^{i \theta_{\mu}(\mathbf{n})} \phi(\mathbf{n}+\hat{\mu}) ;
$$

$T_{\mu}^{\dagger} T_{\mu}=1 ; \quad D^{\dagger}=-D$,

where $\sigma_{\mu}$ are the two-component Pauli matrices. This operator is expected to describe a theory with eight degenerate flavors. Since the staggered-Dirac operator is obtained from the naive-Dirac operator by a change of basis [6,7], it is clear that the spectrum will trivially show a two-flavor degeneracy for all background gauge fields. In addition, for our background gauge field that satisfies Eq. (17), we have a relation similar to the continuum Dirac operator as

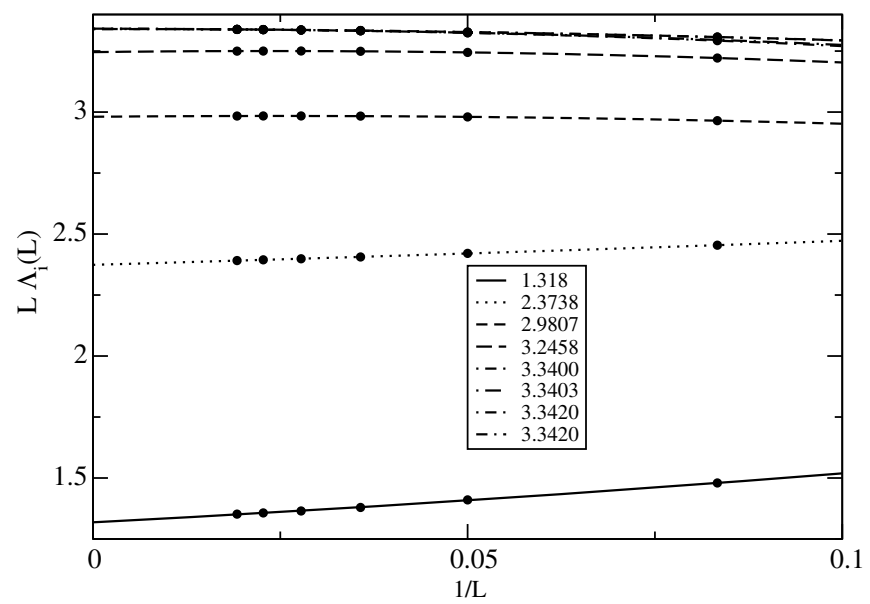

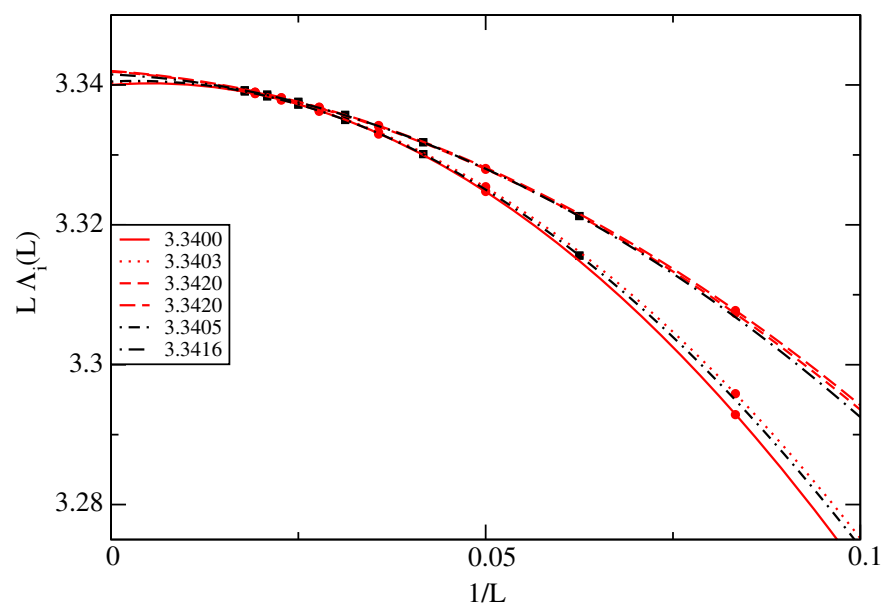

FIG. 2. The low-lying eigenvalues of the naive-Dirac operator as a function of $L$. The top-left plot shows the spectrum for $L=4(2 n+2) ; n=1,2,3,4,5,6$ and shows an eightfold degeneracy. The top-right plot shows the spectrum for $L=4(2 n+1) ; n=1$, $2,3,4,5,6$ and shows a fourfold degeneracy. The bottom plot shows the third and fourth distinct eigenvalues for $L=4(2 n+2)$ (in black) and the fifth to eighth distinct eigenvalues for $L=4(2 n+1)$ (in red). All these different spectral levels in the bottom panel are expected to become degenerate only when $L \rightarrow \infty$. 


$$
\bar{P} \not \bar{P}^{\dagger}=-\not \text {. }
$$

The above parity doubling will lead to at least a fourfold degeneracy of the spectrum of

$$
D^{\dagger} D \psi_{i}=\Lambda_{i}^{2} \psi_{i} ; \quad 0<\Lambda_{1}<\Lambda_{2}<\cdots
$$

If naive-Dirac fermions do not break the flavor symmetry, we should therefore find a 16 -fold degenerate spectrum. We will compute the low-lying eigenvalues of $D^{\dagger} D$ using the Ritz algorithm [21] and impose antiperiodic boundary conditions in one of three directions (we choose the $y$ direction). We expect $\lambda_{i}=\lim _{L \rightarrow \infty} \Lambda_{i} L$ to be finite and nonzero. For reference, the three distinct lowest eigenvalues for free fermions with antiperiodic boundary conditions in one of three directions will be $\left(\lambda_{1}, \lambda_{2}, \lambda_{3}\right)=(1, \sqrt{5}, 3) \pi$. The results for the lowest 32 eigenvalues are shown in Fig. 2. Let us first focus on the top-left plot in Fig. 2, which corresponds to even values of $s$ obtained by setting $L=$ $4(2 n+2)$ for $n=1,2,3,4,5,6$. The first two-lying distinct eigenvalues have an eightfold degeneracy, and the third distinct eigenvalue has an almost 16-fold degeneracy. Therefore, we conclude that the eightfold flavor symmetry is broken into two remnant fourfold flavor symmetries at the lowest level and this effect persists all the way to $L \rightarrow \infty$. When we look at the spectrum in the top-right plot corresponding to odd values of $s$ obtained by setting $L=$ $4(2 n+1)$ for $n=1,2,3,4,5,6$, we see that the four lowlying distinct eigenvalues have only a fourfold degeneracy. Therefore, the flavor symmetry is broken to the minimum required by the trivial twofold symmetry required by the presence of two copies of staggered fermions. Furthermore, this flavor breaking persists all the way to $L \rightarrow \infty$. Focusing on the bottom plot, the third and fourth distinct eigenvalues when $L=4(2 n+2)$ and the fifth to eighth distinct eigenvalues when $L=4(2 n+1)$ all approach a 16-fold degeneracy when $L \rightarrow \infty$, and the results from $L=$ $4(2 n+1)$ and $L=4(2 n+2)$ match. We fitted

$$
\Lambda_{i} L=\lambda_{i}+\frac{\alpha_{i}}{L}+\frac{\beta_{i}}{L^{2}}
$$

using a standard least-square fit, and the fitted values of $\lambda_{i}$ are quoted in Fig. 2 as legends of the corresponding fits. To make the point the 16-fold degeneracy is achieved only when $L \rightarrow \infty$, we have listed the fits from the four fourfold degenerate spectrum for $L=4(2 n+1)$ and the two eightfold degenerate spectrum for $L=4(2 n+2)$ in all three plots. The convergence in the actual data as $L \rightarrow \infty$ is better than what is seen in the fitted values at $L \rightarrow \infty$. We expect any slight disagreement between the almost degenerate extrapolated eigenvalues to be within systematical errors associated with the fit form in Eq. (27).

\section{WILSON-DIRAC FERMIONS}

The Wilson term,

$B-m_{w}=\frac{1}{2} \sum_{\mu=1}^{3}\left(2-T_{\mu}-T_{\mu}^{\dagger}\right)-m_{w} ; \quad B=B^{\dagger}$,

will lift the doublers observed in Sec. III, and

$$
X=B-m_{w}+D ; \quad X^{\dagger}=B-m_{w}-D
$$

are Wilson-Dirac fermions for a pair of two-component fermions related by parity. The mass term is parity even as long as we view $\left(B-m_{w}\right)$ as a whole as the mass term with $m_{w} \in(-2,2)$. We have used an unconventional notation for the sign of the mass to make it convenient for the definition of overlap-Dirac fermions.

The Wilson-Dirac fermion action for a pair of twocomponent fermions that is parity invariant is given by

$$
S_{\mathrm{fw}}=\left(\begin{array}{ll}
\bar{\phi}_{2} & \bar{\phi}_{1}
\end{array}\right)\left(\begin{array}{cc}
0 & X^{\dagger} \\
X & 0
\end{array}\right)\left(\begin{array}{c}
\phi_{1} \\
\phi_{2}
\end{array}\right) .
$$

For our particular background which obeys Eq. (17), we have $\bar{P}^{\dagger} X \bar{P}=X^{\dagger}$, and we can identify $\phi_{2}$ with $\bar{P}^{\dagger} \phi_{1}$. Since we can only discuss the spectrum of a four-component parity invariant fermion, we do not have the double degeneracy present in two-component naive fermions at the expense of removing the doublers. The eigenvalues of the four-component fermion operator come in $\pm \Lambda_{i}$ pairs where $\Lambda_{i}>0$ are obtained from the eigenvalue problem

$$
X^{\dagger} X \psi_{i}=\Lambda_{i}^{2} \psi_{i} ; \quad 0<\Lambda_{1}<\Lambda_{2}<\cdots .
$$

Using Eq. (29), we can write

$$
X^{\dagger} X=-D^{2}+[B, D]+\left(B-m_{w}\right)^{2} .
$$

If we consider gauge field configurations generated by the standard noncompact Wilson action [gauge fields on links will scale as $\frac{1}{L}$ at a fixed $L$ when the background field is set to zero in Eq. (7)] as was done in Ref. [2], we expect $D$ to scale like $\frac{1}{L}$ and $B$ to scale like $\frac{1}{L^{2}}$. To maintain a finite physical mass, we set $m_{w}=\frac{m}{L}$ where we keep $m$ fixed as we take $L \rightarrow \infty$. In this setup, we expect

$$
\lambda_{i}(m)=\lim _{L \rightarrow \infty} L \sqrt{\Lambda_{i}^{2}-m_{w}^{2}}
$$

to be finite and nonzero. Furthermore, we expect $\lambda_{i}(m)$ to be independent of $m$ and consistent with the value obtained using naive-Dirac fermions.

\section{A. Properties at finite physical mass $m=m_{w} L$}

We first set $m_{w}=0$ and plot the four lowest eigenvalues, $L \Lambda_{i}(L)$, as a function of $\frac{1}{L}$ in Fig. 3. The data fit Eq. (27) 


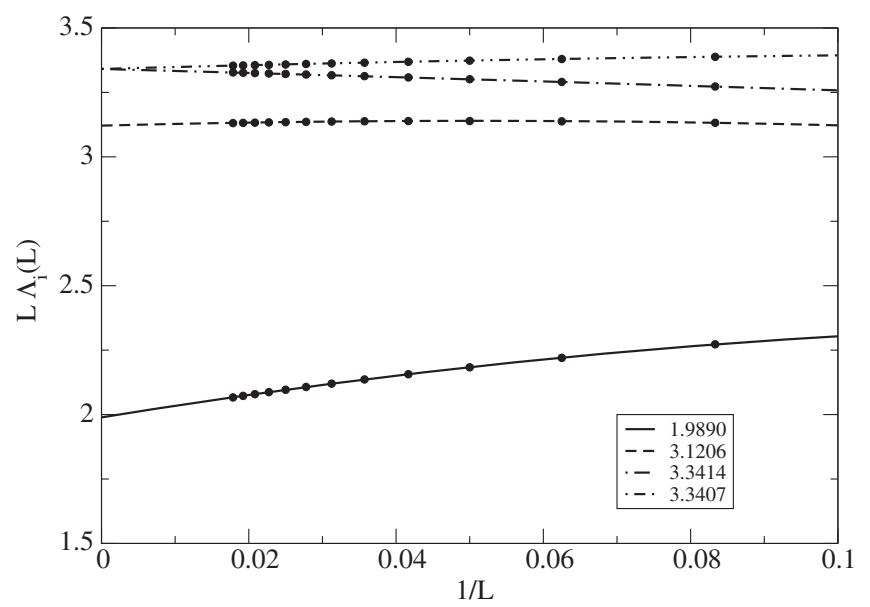

FIG. 3. The low-lying eigenvalues of the Wilson-Dirac operator with $m_{w}=0$ as a function of $L$.

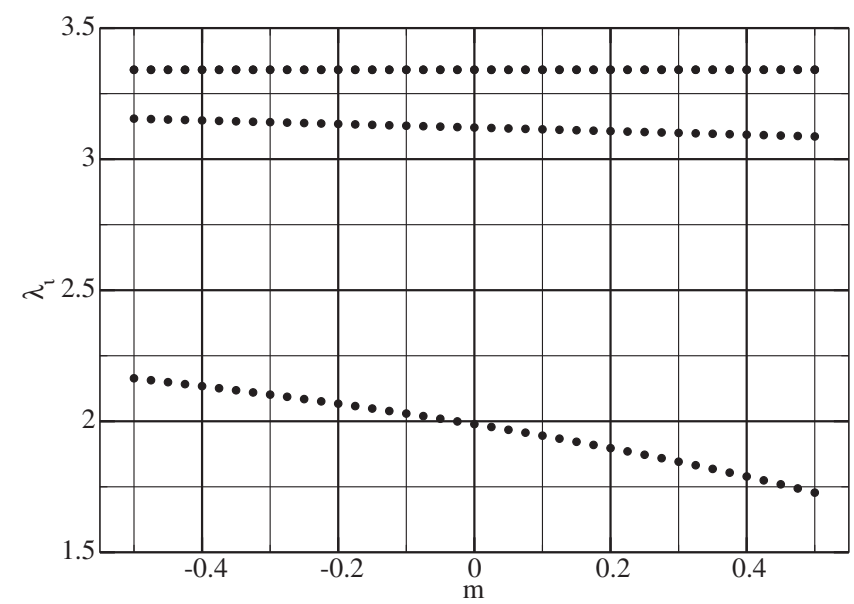

FIG. 4. The low-lying eigenvalues, $\lambda_{i}(m)$ of the Wilson-Dirac operator obtained in the limit of $L \rightarrow \infty$ as a function of $m$.

well, and the fitted values of $\lambda_{i}$ are quoted in Fig. 3 as legends of the corresponding fits. On the one hand, the two lowest eigenvalues approach different limits as $L \rightarrow \infty$, showing that Wilson-Dirac fermions do not recover a double degenerate spectrum realized by naive fermions that satisfies Eq. (25). On the other hand, we see that there is good agreement in the $L \rightarrow \infty$ limit between the two lowest eigenvalues $\left(\lambda_{1}\right.$ and $\left.\lambda_{2}\right)$ for the Wilson-Dirac operator and the two lowest eigenvalues associated with the black lines (case of eightfold degeneracy) in Fig. 2. The doubling seen in the 16-fold degenerate spectrum of naiveDirac fermions in Fig. 2 is also seen in Fig. 3, since $\lambda_{3}$ and $\lambda_{4}$ are equal. Furthermore, the values for $\lambda_{3}=\lambda_{4}$ match well with the corresponding value obtained from naiveDirac fermions. We conclude that naive-Dirac and massless Wilson-Dirac fermions behave in the same manner in the continuum limit with $Q=1$ :

(i) The two lowest eigenvalues show a splitting either due to the breaking of flavor symmetry or due to the need for two different two-component Wilson-Dirac operators to realize a single fermion flavor.

(ii) The rest of the spectrum shows the expected twofold degeneracy per two-component flavor (explicitly seen for the third distinct eigenvalue).

In order to observe possible effects due the Wilson term not being irrelevant, we proceed to study the behavior of the eigenvalues as a function of $m=m_{w} L$. To this end, we plot the first four values of $\lambda_{i}(m)$, obtained by fitting the righthand side of Eq. (33) using Eq. (27), in Fig. 4. We note that $\lambda_{1}(m)$ and $\lambda_{2}(m)$ depends on $m$, suggesting that $B$ and $[B, D]$ do not scale naively as expected. This is an effect of the background as viewed by Wilson-Dirac fermions. But we see that $\lambda_{3}(m)=\lambda_{4}(m)$ are independent of $m$. The effect of a nonsmooth background with $Q=1$ affects only the two lowest eigenvalues even as a function of $m$. Note that naive-Dirac fermions will show the expected quadratic dependence of mass simply because the mass term commutes with $\not D$.

\section{B. Properties at Wilson mass $m_{w}$ that is relevant to the kernel of overlap operator}

Finally, we need to understand the behavior of the lowlying eigenvalues as a function of $m_{w}$ when it is kept fixed as we vary $L$. As long as $m_{w} \neq 0$, it corresponds to a fermion with infinite mass that appears as a kernel for the overlap-Dirac operator. A plot of the four low-lying eigenvalues, $\Lambda_{i}\left(m_{w}\right)$, is shown in the left panel of Fig. 5 for $L=56$, and the effect of a background that is not continuumlike is evident in the behavior of the lowest eigenvalue. The higher eigenvalues seem to show a behavior that reaches a minimum at $m_{w}=0$. The lowest eigenvalue, on the other hand, shows two distinct behaviors for $m_{w}<0$ and $m_{w}>0$. The right panel of Fig. 5 shows that the lowest eigenvalue at a fixed $m_{w}$ decreases with increasing $L$ for $m_{w}>0$, whereas the lowest eigenvalue approaches a nonzero limit at infinite $L$ for $m_{w}<0$. For $m_{w}<0$, the eigenvalue $\Lambda_{1}$ at a fixed $m_{w}$ approaches $m_{w}$ in the $L \rightarrow \infty$ limit, with finite $L$ corrections that are polynomial in $1 / L$. This is similar to the behavior seen in the higher eigenvalues as well. This is shown for a fixed value $m_{w}=-0.275$ in the top-left panel of Fig. 6 where $\Lambda_{1}^{2}$ is plotted as a function of $1 / L$. For $m_{w}>0$, the lowest eigenvalue approaches zero with a distinct $\exp \left(-\beta\left(m_{w}\right) L\right)$ behavior for larger $L$ with an $m_{w}$ dependent coefficient $\beta\left(m_{w}\right)$. This is demonstrated for $m_{w}=0.275$ in Fig. 5 by plotting $\log \left(\lambda_{1}^{2}\right)$ as a function of $L$ where we observe a good description of the large $L$ data by a simple $\exp (-\beta(0.275) L)$ shown by the line. On the other hand, the higher eigenvalues are gapped at finite $m_{w}>0$ for $L \rightarrow \infty$ as we would naively expect. If we examine the dependence of the $\beta\left(m_{w}\right)$ as a function of $m_{w}$, we find $\beta(0)$ is consistent with zero and increases with $m_{w}$ as shown in the bottom panel of Fig. 5. 

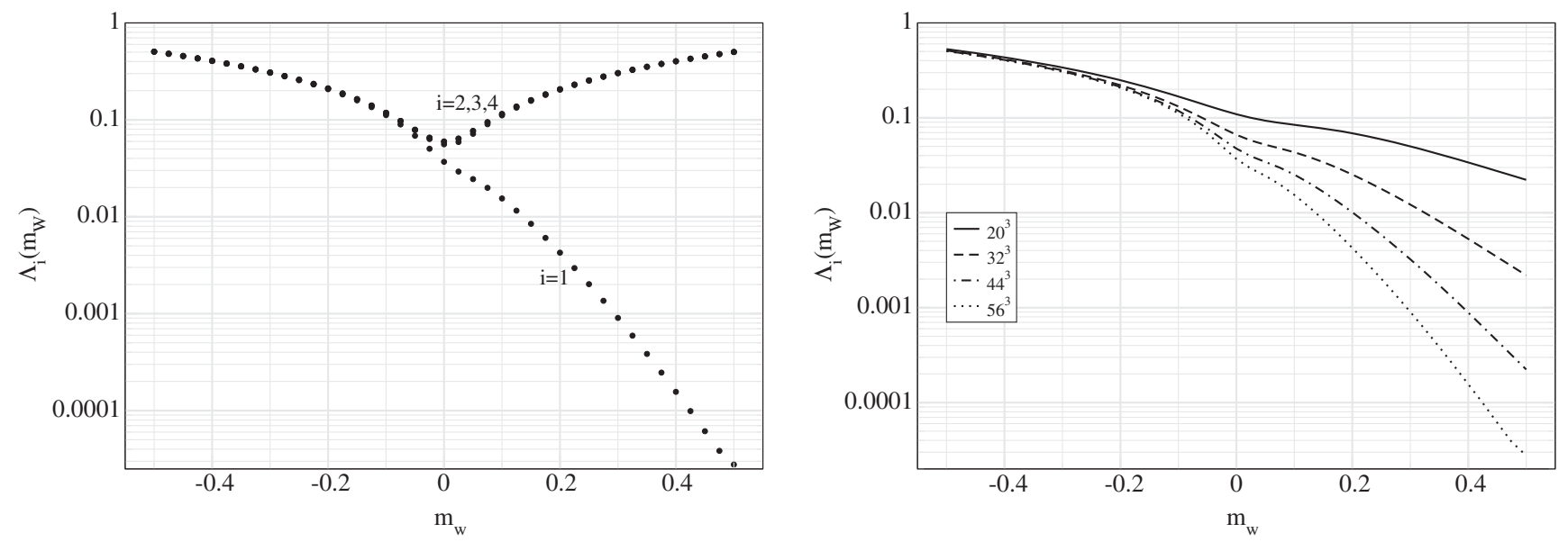

FIG. 5. The low-lying eigenvalues, $\Lambda_{i}\left(m_{w}\right)$, of the Wilson-Dirac operator as a function of $m_{w}$ are shown for $L=56$ in the left panel. The lowest eigenvalue that behaves anomalously for $m_{w}>0$ is shown for four different values of $L$ in the right panel.

We need to study the consequence of the above anomalous behavior of the lowest eigenvalue on the overlap-Dirac operator spectrum where $m_{w}>0$ only plays the role of a regulator and one expects physics to be independent of the choice of $m_{w}$. In addition, the presence of the one anomalously low-lying eigenvalue for positive $m_{w}$ will
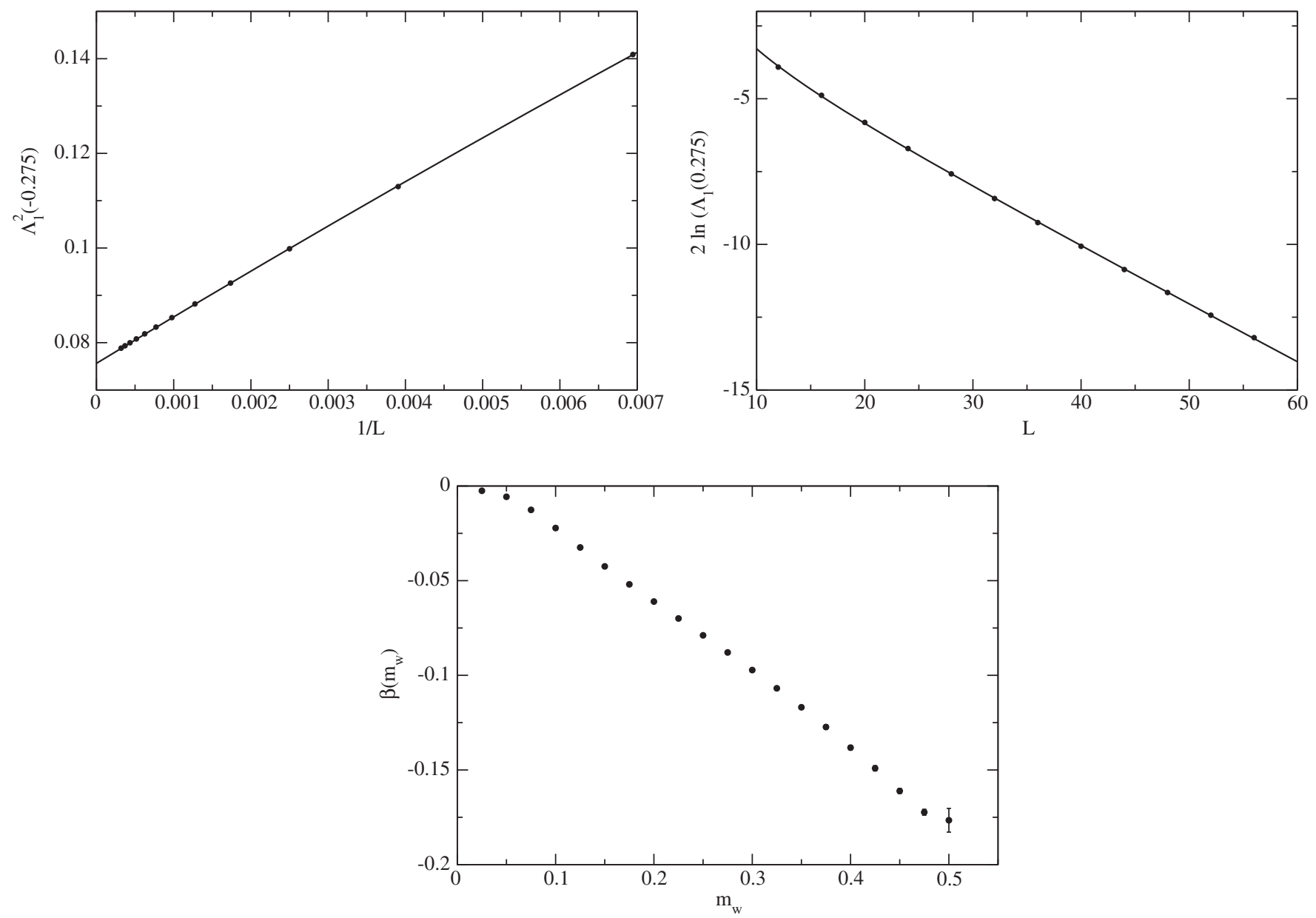

FIG. 6. This plot shows the behavior of the lowest eigenvalue of the Wilson-Dirac operator. In the top-left panel, the approach of $\Lambda_{1}^{2}$ to $m_{w}^{2}$ is shown as a function of $1 / L$ for $m_{w}=-0.275$. In the top-right panel, the exponential decrease of $\Lambda_{1}^{2}$ with an increase in $L$ is shown for $m_{w}=0.275$. In the bottom panel, the $m_{w}$ dependence of $\beta\left(m_{w}\right)$ for the asymptotic exponential decrease $\exp \left(-\beta\left(m_{w}\right) L\right)$ for $m_{w}>0$ is shown. 
affect the numerical computation using the overlap-Dirac operator.

\section{OVERLAP-DIRAC FERMIONS}

The two different two-component massless overlapDirac operators are

$D_{o}=\frac{1+V}{2} ; \quad$ or $\quad D_{o}^{\dagger}=\frac{1+V^{\dagger}}{2} ; \quad V=X \frac{1}{\sqrt{X^{\dagger} X}}$.

Whereas the presence of the Wilson term in the WilsonDirac operator spoiled the commutativity of $X$ and $X^{\dagger}, D_{o}$ commutes with $D_{o}^{\dagger}$. In that sense, overlap-Dirac operator is closer to a continuum Dirac operator- $D_{o}$ cannot be antiHermitian since it has to correctly reproduce the parity anomaly. Since our background field satisfies Eq. (17), the spectrum of $V$ has the following property that results in a double degeneracy in the spectrum of $D_{o}^{\dagger} D_{o}$. Since

$$
\left[\bar{P}^{\dagger} V \bar{P}\right]=V^{\dagger},
$$

we have

$$
V \psi_{j}=e^{i \phi_{j}} \psi_{j} \Rightarrow V\left[\bar{P} \psi_{j}\right]=e^{-i \phi_{j}}\left[\bar{P} \psi_{j}\right],
$$

which will result in a double degeneracy in the spectrum of

$$
D_{o}^{\dagger} D_{o}=\frac{2+V+V^{\dagger}}{4} .
$$

The analysis in Sec. IV has shown the presence of an anomalously small eigenvalue of $X^{\dagger} X$ for $m_{w}>0$. The mass, $m_{w}$, acts as a regulator for overlap-Dirac fermions, and therefore it is natural to study the spectrum of $D_{o}^{\dagger} D_{o}$ as a function of $m_{w}$. Algorithmically, one uses a rational approximation $[22,23]$ of the type

$$
\frac{1}{\sqrt{X^{\dagger} X}}=\sum_{i=1}^{n} \frac{r_{i}}{X^{\dagger} X+p_{i}},
$$

where the values of the residues and poles and the number of them are chosen to approximate the operator on the lefthand side to a desired accuracy in the needed range. This range always has a lower limit away from zero, and the presence of a very small eigenvalue of $X^{\dagger} X$ has to be taken care of by performing

$$
\begin{aligned}
\frac{1}{\sqrt{X^{\dagger} X}} \mathbf{v} & =\frac{1}{\sqrt{\Lambda_{1}}}\left(\mathbf{w}_{1}^{\dagger} \mathbf{v}\right) \mathbf{w}_{1}+\sum_{i=1}^{n} \frac{r_{i}}{X^{\dagger} X+p_{i}}\left(1-\mathbf{w}_{1} \mathbf{w}_{1}^{\dagger}\right) \mathbf{v} ; \\
X^{\dagger} X w_{1} & =\Lambda_{1} w_{1} .
\end{aligned}
$$

With this algorithm in place for numerically dealing with the overlap-Dirac operator, we computed the four low-lying eigenvalues of

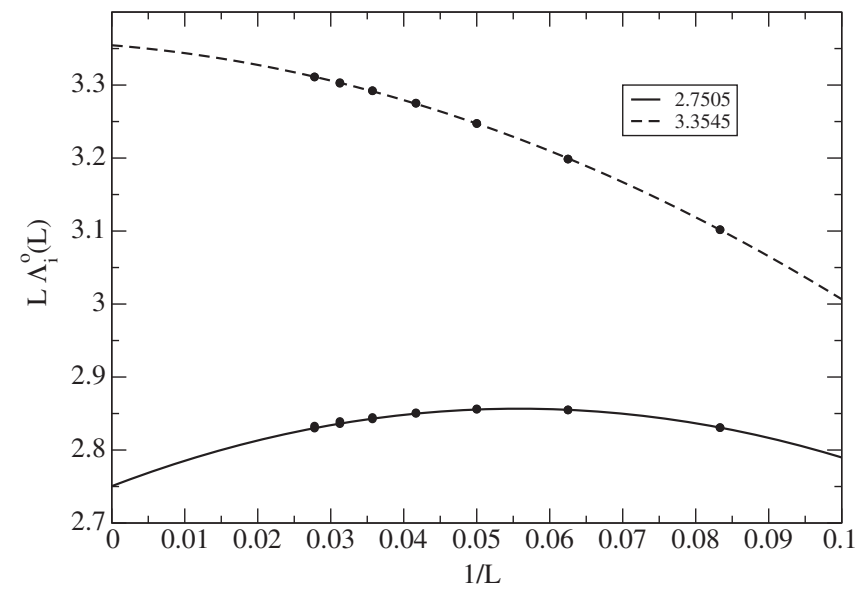

FIG. 7. The two low-lying distinct eigenvalues, $\Lambda_{i}^{o}$, of the overlap-Dirac operator as a function of $L$.

$$
\left[D_{o}^{\dagger} D_{o}\right] \psi_{i}=\left[\frac{\Lambda_{i}^{o}}{2 m_{w}}\right]^{2} \psi_{i}
$$

where we have accounted for the trivial mass renormalization that arises from the mass of the Wilson-Dirac fermion [24]. Due to the fact that the lowest eigenvalue of the Wilson-Dirac operator becomes very small as $L$ is increased, we only went up to $L=36$, where the lowest eigenvalue is still large enough to enable its projection to the desired accuracy. The spectrum clearly comes in degenerate pairs due to Eq. (36). The approach to the infinite $L$ limit of the two low-lying distinct eigenvalues, $\Lambda_{i}^{o}$, is shown in Fig. 7 with $m_{w}=0.425$, where we fitted the data to the form like for naive-Dirac fermions, namely, as in Eq. (27). If we compare with the result for Wilson-Dirac fermions in Fig. 3, we see that there is a reasonable agreement between the second distinct eigenvalue of the massless overlap-Dirac operator and the third distinct eigenvalue of the massless Wilson-Dirac operator that is doubly degenerate. The lowest eigenvalue of the overlap-Dirac operator that also shows a double degeneracy falls in between the two lowest eigenvalues of the Wilson-Dirac operator, and it shows strong finite $L$ effects, but there is no simple relationship between the lowest eigenvalue of the overlap-Dirac operator and the two lowest eigenvalues of the WilsonDirac operator.

Finally, we plot the spectrum of the two low-lying distinct eigenvalues of the massless overlap-Dirac operator as a function of the Wilson-Dirac mass in Fig. 8. Two features are evident. There is clear evidence of a double degeneracy in the spectrum within numerical errors arising from the anomalously small eigenvalue of $X^{\dagger} X$ being not treated accurately enough. The spectrum is essentially independent of $m_{w}$ for $m_{w}>0.3$. If the background configuration were continuumlike, we would have seen an independence on $m_{w}$ over the entire range. 


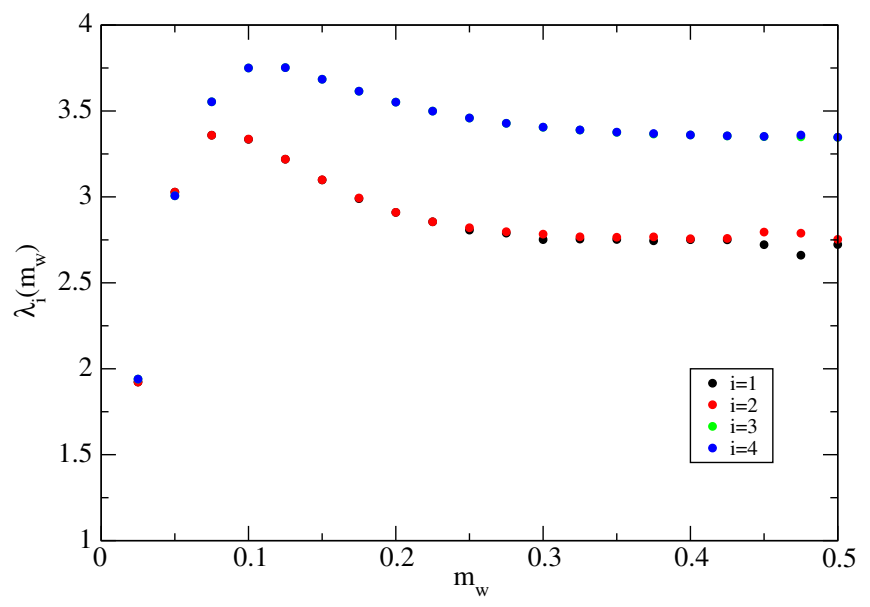

FIG. 8. The two low-lying distinct eigenvalues, $\lambda_{i}^{o}$, of the overlap-Dirac operator as a function of $m_{w}$.

\section{CONCLUSIONS}

We defined a background flux corresponding to a monopole-antimonopole pair separated by a distance $\frac{L}{4}$ on a $L^{3}$ lattice by a noncompact flux of $2 \pi$ units on a single plaquette in the $z$ direction for an extent of $\frac{L}{4}$. Using the standard noncompact Wilson action on the lattice, we found the noncompact link variables that minimizes the action in the presence of the above background. A standard continuum limit does not exist for the gauge field that minimizes the action - the noncompact link variables do not approach zero as we take $L \rightarrow \infty$. This is akin to discretizing a spherical monopole - the link variables on the plaquette surrounding the monopole do not go to zero as we take $L \rightarrow \infty$. The main question we asked in this paper is the following. Let us couple the monopoleantimonopole background to a parity invariant lattice massless fermion action using the compact link variables. How do different versions of lattice regularization show the effect of a background that is not continuumlike?

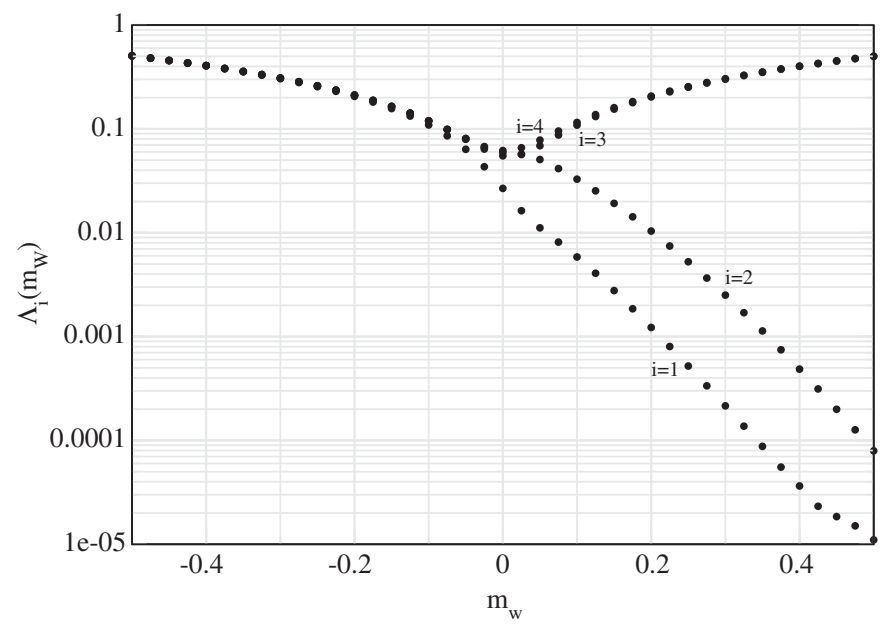

Due to the background gauge field being invariant under a combination of parity and a particular lattice translation given by Eq. (17), we expect the spectrum to be doubly degenerate if the lattice fermion is able to respect this symmetry. The naive-Dirac fermion respects this symmetry but describes eight (four if we reduced it to staggered fermions) fermion flavors. The Wilson-Dirac fermion does not respect this symmetry because the doublers are lifted by realizing the two different two-component fermions related by parity by an operator and its Hermitean conjugate that do not commute. As such, neither the naive-Dirac fermion nor Wilson-Dirac fermion shows a doubly degenerate spectrum at the lowest level for $Q=1$; the 16-fold degeneracy for eight flavors of naive-Dirac fermions is either split into two eightfold or four fourfold degeneracies, implying that flavor symmetry is not realized even when $L \rightarrow \infty$, and the twofold degeneracy for one flavor of Wilson-Dirac fermion is split into two, implying that Wilson-Dirac fermion does not recover the expected degeneracy even when $L \rightarrow \infty$. In spite of this, the spectrum of naive-Dirac fermions and massless Wilson-Dirac fermions match well. The effect of splitting of the lowest twofold degenerate level is also seen in the two lowest eigenvalues of the spectrum of the WilsonDirac operator with a physically finite mass. In addition to this unanticipated behavior, Wilson-Dirac fermion has an anomalously small eigenvalue for one sign of the WilsonDirac mass that realizes a nonzero Chern-Simons term $[15,16]$. Contrary to Wilson-Dirac fermions, the low-lying eigenvalues of the overlap-Dirac show the anticipated twofold degeneracy as long as we have evaluated the action of the overlap-Dirac operator accurately. The spectrum is independent of the Wilson-Dirac mass parameter that appears in the kernel of the overlap-Dirac operator as long as the Wilson-Dirac mass parameter is away from zero.

In spite of the fact that sensible results about monopoles could be obtained using overlap-Dirac fermions, we expect a numerical computation to be difficult. The low-lying

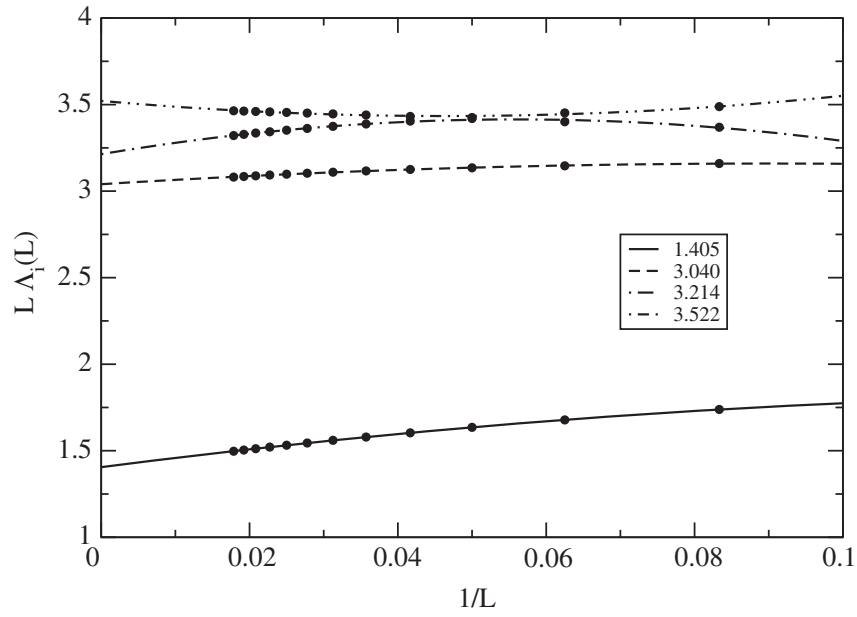

FIG. 9. The low-lying eigenvalues, $\Lambda_{i}\left(m_{w}\right)$, of the Wilson-Dirac operator as a function of $m_{w}$ at $L=56$ for a monopole-antimonopole pair with $Q=2$ are shown in the left panel. The low-lying spectrum at $m_{w}=0$ is shown as a function of $L$ in the right panel. 
eigenvalue(s) of the Wilson-Dirac operator that appears in the kernel of the overlap-Dirac operator will affect the numerical computation. A study of compact QED using overlap-Dirac fermions is possible in principle, but it will be numerically very expensive to study such a theory due to the proliferation of low-lying eigenvalues arising from a finite density of monopoles. This is evident in the left panel of Fig. 9, where the low-lying eigenvalues of the WilsonDirac operator as a function of Wilson-Dirac mass are plotted in the presence of a monopole-antimonopole pair with $Q=2$. There are two anomalously small eigenvalues for $m_{w}>0$. In addition, the splitting of the twofold degenerate spectrum is now seen in the lowest four eigenvalues of the massless Wilson-Dirac operator as shown in the right panel of Fig. 9. Therefore, both anomalous effects increase with $Q$. Yet, we expect the massless overlap-Dirac operator to exhibit proper behavior as long as the numerical evaluation of the operator is performed accurately.
In spite of the anomalous behavior of the low-lying eigenvalues of the Wilson-Dirac operator, the massless operator produced the expected dimension of the monopole operator in Ref. [19]. This is probably due to the fact that the entire spectrum contributes to the dimension of the monopole operator and only the two lowest eigenvalues show a splitting of the twofold degeneracy. Therefore, a cheaper alternative would be to proceed in the same direction and compute the dimension of the monopole operator in noncompact QED using Wilson-Dirac fermions in a fixed monopole-antimonopole background, and a computation in this direction is currently in progress.

\section{ACKNOWLEDGMENTS}

R. N. acknowledges partial support by the NSF under Grant No. PHY-1515446. N. K. acknowledges support by the U.S. Department of Energy under Contract No. DESC0012704.
[1] S. Hands and J. B. Kogut, Nucl. Phys. B335, 455 (1990).

[2] N. Karthik and R. Narayanan, Phys. Rev. D 93, 045020 (2016).

[3] N. Karthik and R. Narayanan, Phys. Rev. D 94, 065026 (2016).

[4] N. Karthik and R. Narayanan, Phys. Rev. D 96, 054509 (2017).

[5] W. Armour, S. Hands, J. B. Kogut, B. Lucini, C. Strouthos, and P. Vranas, Phys. Rev. D 84, 014502 (2011).

[6] J. B. Kogut and L. Susskind, Phys. Rev. D 11, 395 (1975).

[7] C. Gattringer and C. B. Lang, Lect. Notes Phys. 788, 1 (2010).

[8] M. Creutz, Proc. Sci., CONFINEMENT8 (2008) 016 [arXiv:0810.4526].

[9] M. Creutz, Phys. Rev. D 78, 078501 (2008).

[10] M. Creutz, arXiv:0804.4307.

[11] M. Creutz, Proc. Sci., LATTICE2007 (2007) 007 [arXiv: 0708.1295].

[12] H. Neuberger, Phys. Lett. B 417, 141 (1998).
[13] A. Redlich, Phys. Rev. D 29, 2366 (1984).

[14] A. Niemi and G. Semenoff, Phys. Rev. Lett. 51, 2077 (1983).

[15] A. Coste and M. Luscher, Nucl. Phys. B323, 631 (1989).

[16] N. Karthik and R. Narayanan, Phys. Rev. D 92, 025003 (2015).

[17] S. Hands, J. High Energy Phys. 09 (2015) 047.

[18] S. Hands, Phys. Lett. B 754, 264 (2016).

[19] N. Karthik, Phys. Rev. D 98, 074513 (2018).

[20] Y. M. Shnir, Magnetic Monopoles (Springer, New York, 2005).

[21] T. Kalkreuter and H. Simma, Comput. Phys. Commun. 93, 33 (1996).

[22] J. van den Eshof, A. Frommer, T. Lippert, K. Schilling, and H. A. van der Vorst, Comput. Phys. Commun. 146, 203 (2002).

[23] T.-W. Chiu, T.-H. Hsieh, C.-H. Huang, and T.-R. Huang, Phys. Rev. D 66, 114502 (2002).

[24] R. G. Edwards, U. M. Heller, and R. Narayanan, Phys. Rev. D 59, 094510 (1999). 